\title{
Automated Long-Term Scheduling for the SOFIA Airborne Observatory
}

The NASA Stratospheric Observatory for Infrared Astronomy (SOFIA) is a joint US/German project to develop and operate a gyro-stabilized 2.5-meter telescope in a Boeing 747SP. SOFIA's first science observations were made in December 2010. During 2011, SOFIA accomplished 30 flights in the "Early Science" program as well as a deployment to Germany. The new observing period, known as Cycle 1 , is scheduled to begin in 2012. It includes 46 science flights grouped in four multiweek observing campaigns spread through a 13-month span. Automation of the flight scheduling process offers a major challenge to the SOFIA mission operations. First because it is needed to mitigate its relatively high cost per unit observing time compared to space-borne missions. Second because automated scheduling techniques available for ground-based and space-based telescopes are inappropriate for an airborne observatory.

Although serious attempts have been made in the past to solve part of the problem, until recently mission operations staff was still manually scheduling flights. We present in this paper a new automated solution for generating SOFIA long-term schedules that will be used in operations from the Cycle 1 observing period. We describe the constraints that should be satisfied to solve the SOFIA scheduling problem in the context of real operations. We establish key formulas required to efficiently calculate the aircraft course over ground when evaluating flight schedules. We describe the foundations of the SOFIA long-term scheduler, the constraint representation, and the random search based algorithm that generates observation and instrument schedules. Finally, we report on how the new long-term scheduler has been used in operations to date.

Thomas Civeit, USRA

NASA Ames Research Center

SOFIA Program 